\title{
Laminar phase flow for an exponentially tapered Josephson oscillator
}

\author{
Benabdallah, A.; Caputo, J. G.; Scott, Alwyn C.
}

Published in:

Journal of Applied Physics

Link to article, DOI:

$10.1063 / 1.1286161$

Publication date:

2000

Document Version

Publisher's PDF, also known as Version of record

Link back to DTU Orbit

Citation (APA):

Benabdallah, A., Caputo, J. G., \& Scott, A. C. (2000). Laminar phase flow for an exponentially tapered Josephson oscillator. Journal of Applied Physics, 88(6), 3527-3540. https://doi.org/10.1063/1.1286161

\section{General rights}

Copyright and moral rights for the publications made accessible in the public portal are retained by the authors and/or other copyright owners and it is a condition of accessing publications that users recognise and abide by the legal requirements associated with these rights.

- Users may download and print one copy of any publication from the public portal for the purpose of private study or research.

- You may not further distribute the material or use it for any profit-making activity or commercial gain

- You may freely distribute the URL identifying the publication in the public portal

If you believe that this document breaches copyright please contact us providing details, and we will remove access to the work immediately and investigate your claim. 


\title{
Laminar phase flow for an exponentially tapered Josephson oscillator
}

\author{
A. Benabdallah \\ Laboratoire de Mathématiques, Institut National de Sciences Appliquées and Unité C.N.R.S. 6085, B. P. 8, \\ 76131 Mont-Saint-Aignan cedex, France \\ J. G. Caputo ${ }^{\text {a) }}$ \\ Laboratoire de Physique Mathématique et Théorique, Université de Montpellier II and Unité C.N.R.S. 5095, \\ 34095 Montpellier cedex 05, France
}

A. C. Scott

Department of Mathematics, University of Arizona, Tucson, Arizona 85721; Department of Mathematical Modelling, Technical University of Denmark, Lyngby, Denmark

(Received 15 June 1999; accepted for publication 6 April 2000)

\begin{abstract}
Exponential tapering and inhomogeneous current feed were recently proposed as means to improve the performance of a Josephson flux flow oscillator. Extensive numerical results backed up by analysis are presented here that support this claim and demonstrate that exponential tapering reduces the small current instability region and leads to a laminar flow regime where the voltage wave form is periodic giving the oscillator minimal spectral width. Tapering also leads to an increased output power. Since exponential tapering is not expected to increase the difficulty of fabricating a flux flow oscillator, we suggest that this feature should be incorporated in future designs. (C) 2000 American Institute of Physics. [S0021-8979(00)02314-8]
\end{abstract}

\section{INTRODUCTION}

Although the unidirectional flow of flux quanta in long Josephson junctions provides a means for tunable oscillators at frequencies above $100 \mathrm{Ghz}$ with output powers of a few microwatts, the presently observed spectral linewidths $(\delta f / f)$ of about $10^{-5}$ are unacceptably large for many applications. ${ }^{1,2}$ This noisy behavior may be due to irregular dynamics of the fluxon chain ${ }^{3-6}$ inside the device or noise in the lines. To mitigate these effects, it was suggested in Ref. 7 to use an inhomogenous current feed and employ a new design in which the junction width is exponentially tapered, ${ }^{8}$ making the flow of magnetic flux more regular and improving impedance matching to an external load.

Here we confirm these preliminary results along four main points:

(i) We introduce simple analytical models describing a smooth phase flow of the fluxon chain which we refer to as laminar flow ${ }^{9}$ for which the temporal behavior is periodic, therefore giving a minimal spectral width. These models are validated by numerical simulations.

(ii) We show that when the velocity of the fluxon chain is close to 1 , instabilities occur, leading to a chaotic behavior or resonance with one of the cavity modes.

(iii) An extensive study of parameter space demonstrates that the area of irregular behavior of the fluxon chain decreases strongly when the tapering is increased.

(iv) Tapering a standard flux flow device leads to a more rigid fluxon chain which behaves periodically in time.

These new results indicate that exponentially tapering a Josephson flux flow oscillator will substantially improve its performance.

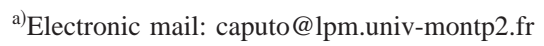

The article is organized as follows. We present the model and recall some preliminary results of Ref. 7 in Sec. II. In Sec. III the laminar flow solution is introduced, leading to simple analytic expressions that we use as benchmarks. Section IV presents numerical computations for the cases of a small and large load, relating these results to the benchmarks of Sec. III. A variety of numerical results are given in Sec. V, including: (i) a study of the resonances that appear near threshold, (ii) the boundary of laminar flow in parameter space, (iii) the influence of exponential tapering on power output and spectral purity, (iv) the influence of exponential tapering on the spectral purity of a standard flux flow oscillator with an external magnetic field and transverse bias current, and (v) the effects of surface current damping on the foregoing results. Concluding remarks are presented in Sec. VI.

\section{EXPONENTIALLY SHAPED JOSEPHSON JUNCTION}

The Josephson junction discussed in this article is shown in Fig. 1. The top panel presents a transverse cut (section $\mathrm{A}-\mathrm{A}^{\prime}$ ) along the direction normal to the junction plates while the bottom panel shows a top view displaying the exponential tapering of the junction width $w(x)=w_{0} e^{-\lambda x}$. The oxide layer separating the two superconducting plates is thicker at the left end of the device realizing an idle region.

Measuring distance and time in appropriate units (the Josephson penetration distance and the reciprocal of the junction plasma frequency ${ }^{4}$ ), the averaged superconducting phase difference $(\varphi)$ in the junction area is described by the following sine-Gordon equation: ${ }^{7,10}$ 


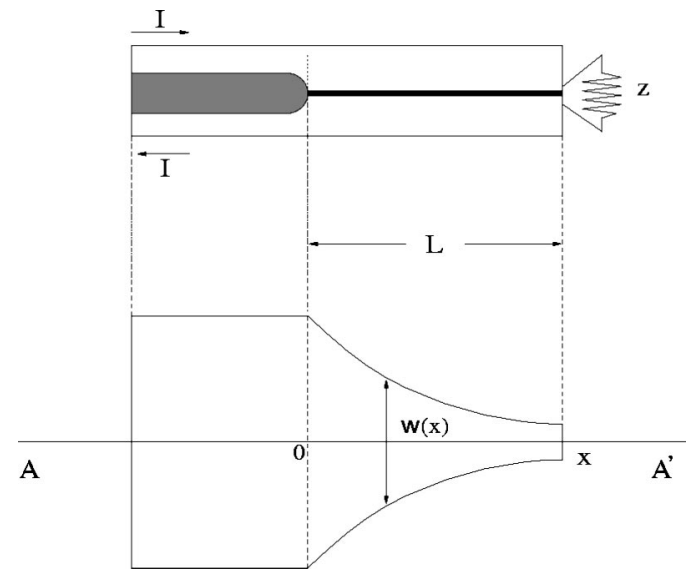

FIG. 1. An exponentially shaped junction together with its idle region and an external load $z$. The top panel is a cut across the junction while the bottom panel is a top view of the device.

$\frac{\partial^{2} \varphi}{\partial t^{2}}-\left(1+\beta \frac{\partial}{\partial t}\right) \frac{\partial^{2} \varphi}{\partial x^{2}}+\sin \varphi+\lambda\left(1+\beta \frac{\partial}{\partial t}\right) \frac{\partial \varphi}{\partial x}+\alpha \frac{\partial \varphi}{\partial t}=0$,

where it is assumed that the external currents and magnetic fields only induce a phase gradient along the $x$ direction. This equation is derived from the condition that the algebraic sum of all the currents at a given point in the junction is zero (Kirchhoff's current law). Reading from the left, the terms are the capacitive current between the plates, the surface current, and its damping, ${ }^{11}$ the Josephson current, the current due to the tapering, and the dissipation due to the normal electrons tunneling across the junction. From Ohm's law, the boundary conditions applied at each end $x=0, L$ of the device are

$$
\begin{aligned}
& \left.\left(1+\beta \frac{\partial}{\partial t}\right) \frac{\partial \varphi}{\partial x}\right|_{x=0}=\eta-I, \\
& \left.\left(1+\beta \frac{\partial}{\partial t}\right) \frac{\partial \varphi}{\partial x}\right|_{x=L}=\eta-\left.\frac{1}{z} \frac{\partial \varphi}{\partial t}\right|_{x=L},
\end{aligned}
$$

where $\eta$ is an external magnetic field, $I$ is an external bias current, and $z$ is the impedance of the load representing the connection of the junction to a microwave line. Throughout this article we have chosen to neglect surface damping by setting $\beta=0$. This assumption is justified in Sec. IX; thus Eqs. (2.1)-(2.3) reduce to

$$
\frac{\partial^{2} \varphi}{\partial t^{2}}-\frac{\partial^{2} \varphi}{\partial x^{2}}+\sin \varphi+\lambda \frac{\partial \varphi}{\partial x}+\alpha \frac{\partial \varphi}{\partial t}=0
$$

and

$$
\left.\frac{\partial \varphi}{\partial x}\right|_{x=0}=\eta-I,\left.\quad \frac{\partial \varphi}{\partial x}\right|_{x=L}=\eta-\left.\frac{1}{z} \frac{\partial \varphi}{\partial t}\right|_{x=L} .
$$

This is the usual sine-Gordon model for long Josephson junctions apart from the $\lambda \partial \varphi / \partial x$ term which corresponds to a geometrical force driving the fluxons towards the right. ${ }^{4}$

Assuming $\eta=0$, we showed in Ref. 7 that at most a half fluxon static solution exists for Eq. (2.4) and that this static solution ceases to exist when $I$ exceeds the threshold value

$$
I_{\theta}=2(1-\lambda) .
$$

Thus for $I<I_{\theta}$ the solution of Eq. (2.4) is static, but for $I$ $>I_{\theta}$ the solution is time dependent, $V>0$, and flux flow is observed.

An important feature of Eq. (2.4) is that any traveling wave solution of the pure sine-Gordon equation with the velocity

$$
u=u_{m}=\frac{\lambda}{\alpha}
$$

is also a solution of Eq. (2.4). ${ }^{7}$ Noting that the speed of flux flow is

$$
u=-\frac{\varphi_{t}}{\varphi_{x}}
$$

(where the subscripts indicate partial derivatives), a matched impedance load for the oscillator is evidently

$$
z_{m}=\frac{\lambda}{\alpha} .
$$

Since $\lambda / \alpha$ fixes the velocity of flux flow in the interior of the device while $z$ determines the velocity at the right hand end, the relative values of these two parameters will be important in distinguishing different types of behavior. If their values greatly differ, the solution of Eq. (2.4) will attempt to satisfy both velocity conditions, leading to a disruption of laminar flow. Note also that the value $\lambda / \alpha=1$ is critical for these parameters because as we will show below, a kink will develop for this velocity and perturb the flux flow.

To numerically integrate Eq. (2.4), we have transformed it into a system of coupled first order equations that are solved by the method of lines, ${ }^{12}$ using an ordinary differential integrator to advance in time while the spatial part was discretized via centered finite differences. In this article, results are presented for a junction of length $L=10$ with 600 points, but in many cases the computations were checked by doubling this number. The damping coefficient was taken to be $\alpha=0.05$ throughout the study. ${ }^{13}$

\section{LAMINAR FLOW OPERATION}

Before presenting the numerical results, we describe the concept of laminar flow of flux quanta from the current bias source $(I)$ into the load and use it to obtain benchmarks for the calculations. A close approximation to laminar flow is shown in Fig. 2, which displays numerical solutions of Eqs. (2.4) and (2.5) with the parameter values

$$
z=2, \quad \lambda / \alpha=0.4, \quad I=3, \quad \text { and } \quad \eta=0
$$

at four different times separated by intervals of $\Delta t=2.5$. Also drawn there is the Laminar flow benchmark (LFL) (see below). With these parameter values, it is seen that $\varphi$ is a smooth monotone function of $x$ which is very well approximated by the benchmark. The relative gradient ratio $\left(\partial \varphi / \partial x-\partial \varphi /\left.\partial x\right|_{\mathrm{LFL}}\right) / \partial \varphi /\left.\partial x\right|_{\mathrm{LFL}}$ varies by about $\pm 10 \%$. Neglecting the small variations in $\partial \varphi / \partial x$ defines the phenomenon of laminar flow. 

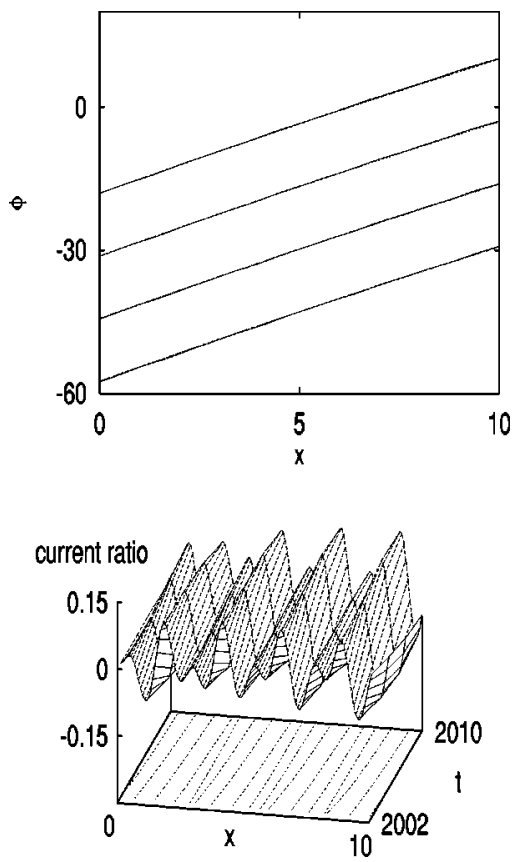

FIG. 2. Phase $\varphi$ as a function of $x$ for four successive times (top panel) with corresponding gradient $\partial \varphi / \partial \mathrm{x}$ normalized by the LFL benchmark as a function of $x$ and $t$ (bottom panel). In the top panel the numerical solution is drawn as a dashed line and the LFL benchmark as a solid line. Note that the phase is decreasing with time. In the bottom panel the zero value contour lines are drawn as dashed. The device parameters are $z=2, \lambda / \alpha=0.4$, $I=3$, and $\eta=0$.

Assuming laminar flow suggests two limiting cases or numerical benchmarks that are described in this section. The first is the high voltage limit which arises when the junction voltage $V=\partial \varphi / \partial t$ is sufficiently large that the time average of $\sin \varphi$ in Eq. (2.4) is effectively zero. The second, called the linear flow limit, assumes that $\partial \varphi / \partial x$ is a linear function of $x$.

\section{A. The high voltage limit (HVL)}

The behavior observed in Fig. 2 is typical of larger values of $z$ and $\lambda / \alpha$. Then the fluctuations of the instantaneous voltage $\partial \varphi / \partial t$ in both $x$ and $t$ are small enough to be neglected to a first approximation, leading to laminar phase flow. In order to simplify the derivation, we choose $\eta=0$, but the calculations can also be carried out in that case.

Assuming that the voltage $V$ is independent of $x$ and $t$ and averaging Eq. (2.4) over time, we obtain

$$
\frac{\partial^{2} \varphi}{\partial x^{2}}=\lambda \frac{\partial \varphi}{\partial x}+\alpha V .
$$

In order for the voltage to be greater than zero (in other words for the flux to move) it is necessary that the bias current satisfy the threshold condition

$$
I>I_{\theta},
$$

where $I_{\theta}$ is defined in Eq. (2.6). Thus in the HVL approximation, appropriate boundary conditions are

$$
\frac{\partial \varphi}{\partial x}(0, t)=-I, \quad \frac{\partial \varphi}{\partial x}(L, t)=-\frac{V}{z} .
$$

This is a linear system that is readily solved for

$$
V \doteq \frac{\lambda e^{\lambda L} z I}{\lambda+\alpha z\left(e^{\lambda L}-1\right)},
$$

from which the power into the load $(z)$ is

$$
P \doteq z\left[\frac{\lambda I e^{\lambda l}}{\lambda+\alpha z\left(e^{\lambda L}-1\right)}\right]^{2}
$$

and the corresponding velocity of the flux helix is

$$
v=-\frac{V}{\varphi_{x}} \doteq \frac{\lambda z}{(\lambda-\alpha z) e^{\lambda(x-L)}+\alpha z} .
$$

\section{B. Linear flow limit (LFL)}

Another way of approximating laminar flow is to use a variational approach where $\partial \varphi / \partial x$ is assumed to be a linear function of $x$. The variational analysis, which is based on conservation of the energy by the sine-Gordon Hamiltonian, is carried through in Appendix A. The result of this analysis is that for $I>I_{\theta}$ the current-voltage $(I-V)$ characteristic is given by

$$
V \doteq \frac{(2+\lambda L) z I}{2+2 \alpha L z-\lambda L},
$$

from which the power output is

$$
P \doteq z\left[\frac{(2+\lambda L) I}{2+2 \alpha L z-\lambda L}\right]^{2}
$$

and the traveling wave speed of the flux is

$$
v \doteq \frac{(1+\lambda L / 2) z}{(\lambda-\alpha z) x+1+\alpha L z-\lambda L / 2} .
$$

The value of $\varphi$ computed from this analysis has been plotted on the top panel of Fig. 2 in dashed line. It is in excellent agreement with the numerical value.

\section{Comparison of HVL and LFL approximations}

To better understand the numerical results presented in the following sections, it is important to keep in mind the relationship between the current-voltage expressions in the high voltage limit (HVL) and the linear flow limit (LFL) that are given by Eqs. (3.1) and (3.4).

First of all, we note two conditions under which the HVL and LFL are identical.

(1) In both of these approximations it is assumed that $V$ $=0$ for $I<I_{\theta}$.

(2) For $I>I_{\theta}$ and $\lambda=0$ (no exponential tapering of the junction), both Eqs. (3.1) and (3.4) reduce to

$V \doteq \frac{z I}{1+\alpha L z}$.

For $\lambda L \gg 1$, on the other hand, Eq. (3.1) — for HVLreduces to

$$
V \doteq \frac{\lambda / \alpha}{1-\exp (-\lambda L)} I,
$$

whereas Eq. (3.4)—for LVL—reduces to 

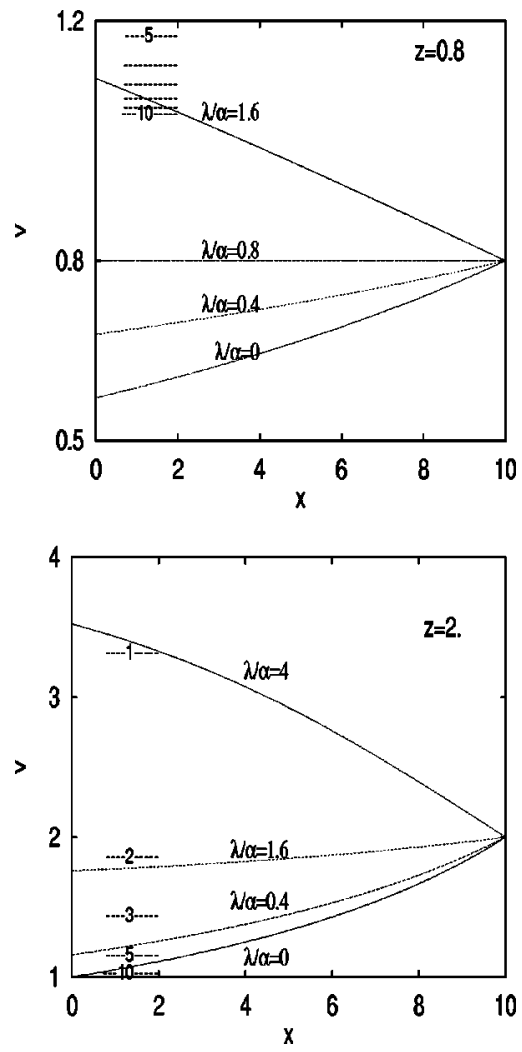

FIG. 3. The velocity of the HVL model from Eq. (3.6) as a function of $x$ for $z=0.8$ (top panel) and $z=2$ (bottom panel) and different values of $\lambda / \alpha$. The phase velocities of the linear modes are plotted on the left hand side together with their index.

$$
V \doteq \frac{2+\lambda L}{2 \alpha L} I
$$

In deciding which of these two benchmarks is more appropriate, recall that the HVL allows a nonlinear dependence of $\varphi_{x}$ upon $x$, whereas the LFL does not ignore the presence of the $\sin \varphi$ term. Thus we can draw two conclusions:

(1) Since it allows for nonlinear dependence of $\varphi$ upon $x$, we expect the HVL limit to be a better benchmark for $\lambda L \gg 1$.

(2) The LFL, on the other hand, will be preferred for $z \gg 1$, because the current $\varphi_{x}$ is reduced in this parameter range, increasing the importance of the $\sin \varphi$ term.

Examples of the utility of these benchmarks are presented in the following sections.

\section{LOADING EFFECTS}

In this section, we consider the effects of varying the load resistance $z$ upon the operation of an exponentially tapered flux flow oscillator. The benchmarks defined above are useful to understand the combined effects of the load resistance $z$ and the tapering exponent $\lambda$. In Fig. 3, we plot the velocity of the HVL model as a function of $x$ for two values of the resistance $z=0.8$ and 2 . As expected for a given $z$, an increase of $\lambda$ causes an increase of the velocity and vice versa. Notice also that the velocity is fixed by the right hand side boundary condition. In the top panel, the value $\lambda / \alpha$
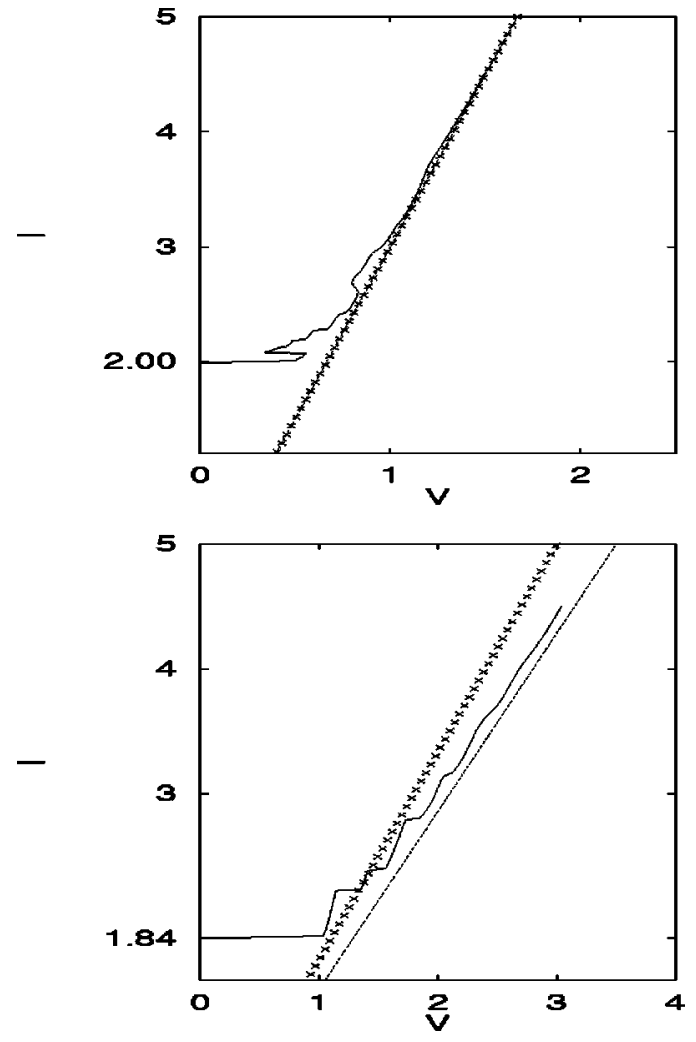

FIG. 4. $I-V$ characteristic curves for $z=0.4$ and $\lambda / \alpha=0$ (top) and 1.6 (bottom). The benchmarks are indicated as: HVL $(* * * * * * * * *)$ and LFL $(\cdots \cdots)$.

$=0.8$ corresponds to the adapted flow (i.e., $z=\lambda / \alpha$ ) so that the velocity is constant. This feature will be seen in some of the simulations.

In Fig. 3 we indicate the phase velocities $v_{n}$ $=\sqrt{1+1 / k_{n}^{2}}$ of the linear cavity modes $k_{n}=n \pi / L$ where we have neglected the influence of $\lambda$. For example $n=2$ corresponds to the mode whose wave number is $k_{2}=2 \pi / L$ and so on. It is expected from the nonlinearity that resonances will occur when the velocity of the fluxon chain is equal to the phase velocity of one of the cavity modes. The quantities $v_{n}$ tend to 1 when $n \rightarrow \infty$ so most of the disturbances of the fluxon chain will happen in the transition region when the velocity goes above 1 . Looking again at Fig. 3, we see that for $z=0.8$ we do not expect resonances for small values of $\lambda / \alpha$ for which the velocity remains small. When $z=2$ these small values of $\lambda / \alpha$ will cause resonances. In actual experiments the junction impedance is small compared with the neighboring wave guide so that $z$ is large. Then tapering the junction will suppress unwanted resonances. A detailed study of these resonances for the case of an infinite load is given in Appendix B.

\section{A. Small external load}

Here we consider the case of a small external load: $z$ $<1$. As mentioned above, this is not experimentally realistic but the example is of pedagogical interest. The currentvoltage characteristic curves are presented in Fig. 4 for $z$ $=0.4$ and $\lambda / \alpha=0$ (top panel) and 1.6 (bottom panel). The laminar flow models give a good description for $\lambda / \alpha=0$ and 
also for $\lambda / \alpha=0.4$ and 0.8 which are not presented. The I-V curve for $\lambda / \alpha=1.6$ presents some resonances at $V$ $=3 \pi / L, 4 \pi / L, 5 \pi / L$, and $6 \pi / L$ as expected from Fig. 3. For a current $I=3.1$ a plot of the traveling traveling wave speed $v=-\varphi_{t} / \varphi_{x}$ as a function of $x$ shows three irregular oscillations around the laminar flow behavior. The laminar flow voltage is not far from that of the $\mathrm{I}-\mathrm{V}$ curve.

For $z=0.8$ the I-V curve is shown in Fig. 5 for $\lambda / \alpha$ $=0.4$ (top panel) and 1.6 (bottom panel). Notice the hysteresis in the curve for $\lambda / \alpha=0.4$ and the clearly marked resonances $V_{n}=n \pi / L$ for $\lambda / \alpha=1$.6. For both values of $\lambda / \alpha$ and for $\lambda / \alpha=0$ and 0.8 (which are not presented), the laminar flow models do not agree with the I-V curve for $I<6$. We expect this since $z=0.8$ forces the speed $v$ of the fluxon chain at the right hand boundary to be close to 1 which is a critical value for which the helical solution of the sineGordon equation breaks down (see below). Figure 6 shows the relative traveling wave speed ratio $\left(v-v_{\mathrm{LFL}}\right) / v_{\mathrm{LFL}}$ for the two plots of Fig. 5 and $I=3$ as a function of $x$ and $t$, where the speed $v=-\varphi_{t} / \varphi_{x}$ has been computed from the numerical solution and $v_{\mathrm{LFL}}$ is the LFL benchmark speed given by Eq. (3.6). For $\lambda / \alpha=0.4$ the speed ratio is smaller than $20 \%$ indicating that the benchmark provides a good aproximation to the solution. A closer view shows strong oscillations of $v$ for $0 \leqslant x \leqslant 5$ which reduce as $5 \leqslant x \leqslant 10$ and $v$ tends to its asymptotic value 0.8 . On the other hand $v_{\text {LFL }}$ increases gradually throughout the junction. For $\lambda / \alpha=1.6$ the benchmark is not a good description because the maximum amplitude of the speed ratio is about $50 \%$, it is negative for $x \leqslant 4$ where the LFL benchmark $v_{\mathrm{LFL}}>1$ and $v<1$. Notice also the strong oscillations occuring near the right end of the junction, there $\varphi_{x}$ becomes small indicating internal degrees of freedom of the fluxon chain as shown in the next section.

\section{B. Stability of phase flow}

The results presented above show that instabilities and resonances occur when the velocity of the fluxon chain reaches 1 . One can understand this effect qualitatively using arguments developed for an infinite medium neglecting the effects of $\lambda$ and $\alpha$. This is meaningful in our situation when there are many oscillations of $\varphi$ in the device and for small $\lambda$ and $\alpha$.

Assuming a propagating solution $\varphi=f(\theta) \equiv f(x-v t)$ of the sine-Gordon equation we obtain after integration

$$
\left(\frac{d f}{d \theta}\right)^{2}=2 \frac{A-2 \sin ^{2}(f / 2)}{v^{2}-1},
$$

where $A$ is an integration constant.

Four different types of solutions can be found depending on $A$ and $v,{ }^{6}$ two oscillating waves around 0 (type 2 in Ref. 6 where $0<A<2$ and $v^{2}<1$ ) and $\pi$ (type $10<A<2, v^{2}$ $>1$ ) and two helical waves for which $d f / d \theta$ does not change sign. These can be subluminous (type $4 A<0, v^{2}$ $<1$ ) or supraluminous (type $3, A>2$ and $v^{2}>1$ ). The case $A=0$ (resp. 2) corresponds to a kink (resp. tachyon). Both
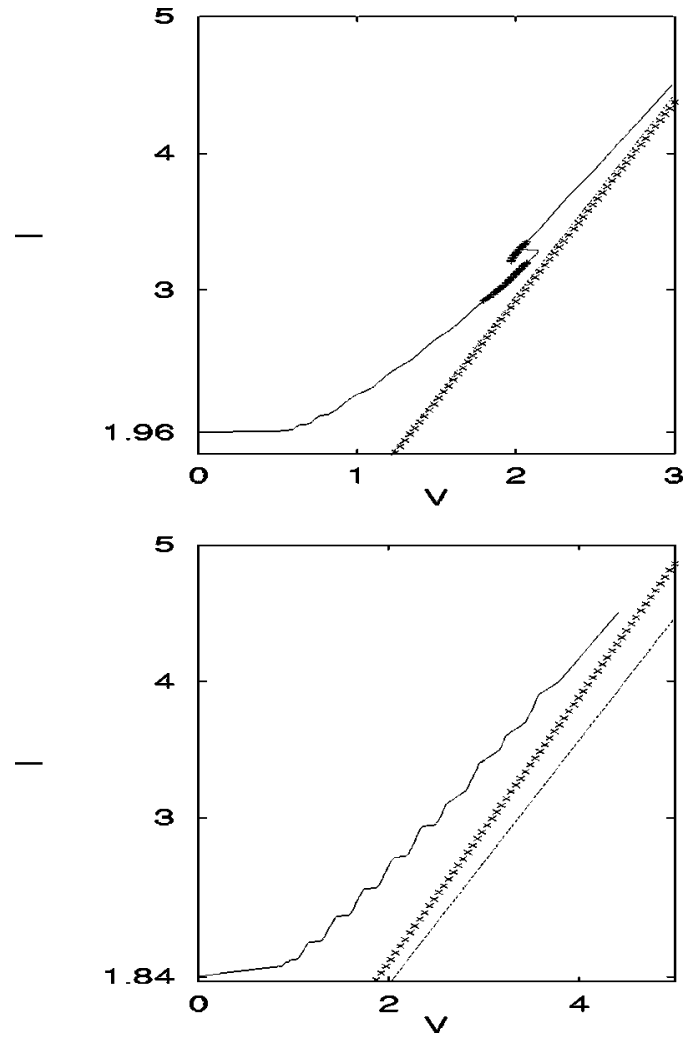

FIG. 5. $I-V$ characteristic $z=0.8, \lambda / \alpha=0.4$ (top), and 1.6 (bottom). The benchmarks are indicated as: HVL $(* * * * * * * *)$ and LFL $(\cdots \cdots \cdots)$.

nonlinear periodic waves are modulationally unstable as opposed to the helical waves, ${ }^{6}$ however for our analysis we are interested in their period.

Let us first consider the subluminous solutions. From Eq. (4.1) one can write $\theta$ as
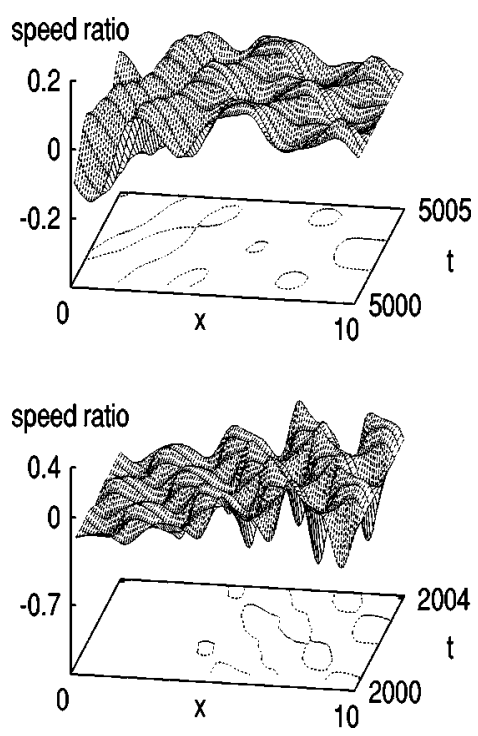

FIG. 6. Plot of the relative traveling wave speed ratio $\left(v-v_{\mathrm{LFL}}\right) / v_{\mathrm{LFL}}$ for the two plots of Fig. 5 and $I=3$ as a function of $x$ and $t$, where the speed $v=-\varphi_{t} / \varphi_{x}$ and $v_{\text {LFL }}$ is the LFL benchmark speed given by Eq. (3.6). The top panel corresponds to $\lambda / \alpha=0.4$ and the bottom panel to 1.6. The zero value contour lines are shown as dashed. 


$$
\theta=-\sqrt{\frac{1-v^{2}}{2(2-A)}} \int^{\pi / 2-\varphi} \frac{d u}{\sqrt{1-\frac{2}{2-A} \sin ^{2} u}},
$$

so that

$$
\varphi=\frac{\pi}{2}-\operatorname{am}\left(-\sqrt{\frac{2(2-A)}{1-v^{2}}} \theta \mid \sqrt{\frac{2}{2-A}}\right),
$$

where am is the amplitude elliptic function. ${ }^{14}$ One can deduce

$$
\frac{d \varphi}{d \theta}=\sqrt{\frac{2(2-A)}{1-v^{2}}} d n\left(-\sqrt{\frac{2(2-A)}{1-v^{2}}} \theta \mid \sqrt{\frac{2}{2-A}}\right),
$$

which is a function of period

$$
T_{4}=2 \sqrt{\frac{2(2-A)}{1-v^{2}}} K\left(\sqrt{\frac{2}{2-A}}\right),
$$

where $K(k)$ is the complete elliptic integral of the first kind of module $k$.

Similarly one can deduce the period for the supraluminous solution

$$
T_{3}=2 K\left(\sqrt{\frac{2}{A}}\right),
$$

and for the two types of non linear periodic waves which are given by the $c n$ elliptic function

$$
\begin{aligned}
& T_{1}=\frac{8}{A} K\left(\sqrt{\frac{A}{2}}\right), \\
& T_{2}=\frac{8 \sqrt{2}}{\sqrt{(2-A)\left(1-v^{2}\right)}} K\left(\sqrt{\frac{2}{A}}\right) .
\end{aligned}
$$

The transition from a subluminous helical wave (type 4 $\left.A<0 v^{2}<1\right)$ to a supraluminous one $\left(A>2 v^{2}>1\right)$ is such that one goes through the intermediate stages $0<A<2$ and $v^{2}<1$ or $v^{2}>1$ corresponding to the nonlinear periodic waves 1 and 2. The value $A=0$ (resp. 2) corresponds to a kink (resp. tachyon) for which $T_{4}$ and $T_{1}$ (resp. $T_{3}$ and $T_{2}$ ) are infinite. The fact that the period of the wave becomes very large implies for our bounded device that the wave will couple to many of the low frequency cavity modes, causing resonances and instabilities. This will cease when the speed of the helical wave is greater than 1 and $A>2$ because then the period will not be large. Therefore the unstable region for the flux flow device corresponds to a speed $v$ close to 1 .

These arguments can be applied to the case shown in Fig. 6 by estimating $A=(1 / 2) \partial \varphi / \partial t^{2}-(1 / 2) \partial \varphi / \partial x^{2}+(1$ $-\cos \varphi$ ) from the simulations. For $\lambda / \alpha=0.4$ (top panel) the average $A<0$ so we have a subluminous helical wave. When $\lambda / \alpha=1.6$ (bottom panel) $-4<A<2$ so the solution alternates between a subluminous helical wave and periodic waves both subluminous and supraluminous, and we find as expected, resonances with the cavity modes in the I-V curve together with a complicated temporal behavior.

Figure 7 shows the formation of a kink in the helical wave. It presents the phase $\varphi(x)$ for 4 successive times for
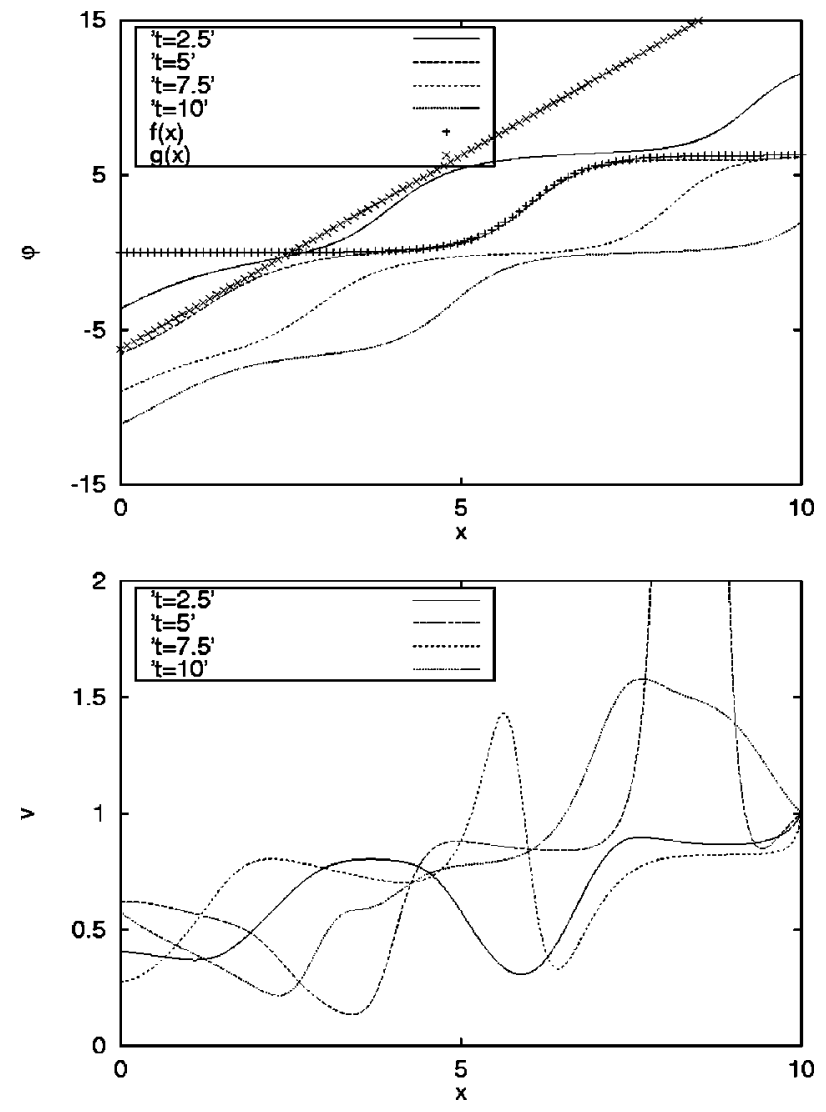

FIG. 7. Plot of the phase $\varphi$ (top) and its corresponding traveling wave speed $-\varphi_{t} / \varphi_{x}$ (bottom) as a function of $\mathrm{x}$ for $z=\lambda / \alpha=1, I=2.1$ for four successive times. For $t=5$, a least square fit with a kink is shown as $f(x)(+)$ and a linear least square as $g(x)(\times)$.

$z=\lambda / \alpha=1$ and a current $I=2.1$ in the top panel and the corresponding speed $v$ in the bottom panel. For $t=2.5$ the speed is everywhere smaller than 1 and no kink is present in the junction. For $t=5$ a kink has formed at $x \approx 6$ as evidenced by the least square fit. The velocity becomes illdefined because $\varphi_{x}$ crosses 0 . Notice how there are no other kinks in the junction. For $t=7.5$ the kink has been annihilated at the right boundary and another one is forming at $x$ $\approx 5$. At $t=10$ the kink has formed and has moved to the right. To summarize, this complicated behavior has nothing to do with the laminar flow models which predict a uniform speed $v \equiv 1$ across the junction.

\section{Large external load}

We now consider the case of a large external load, in which case we expect instabilities for small values of $\lambda / \alpha$ when the speed of the helical wave is around 1 . I-V characteristic curves for $z=2$ are presented in Fig. 8 for $\lambda / \alpha$ $=0,0.4,4$, and 1.6 clockwise. For $\lambda / \alpha=0$ resonances occur at $V_{n}=n \pi / L$ as the helical wave couples with each cavity mode. The hysteretic behavior is due to the fact that as the current is decreased the helical wave does not resonate with the same mode. As $\lambda / \alpha$ is increased from 0.4 to 1.6 the wave travels faster and less resonances occur. These disappear 

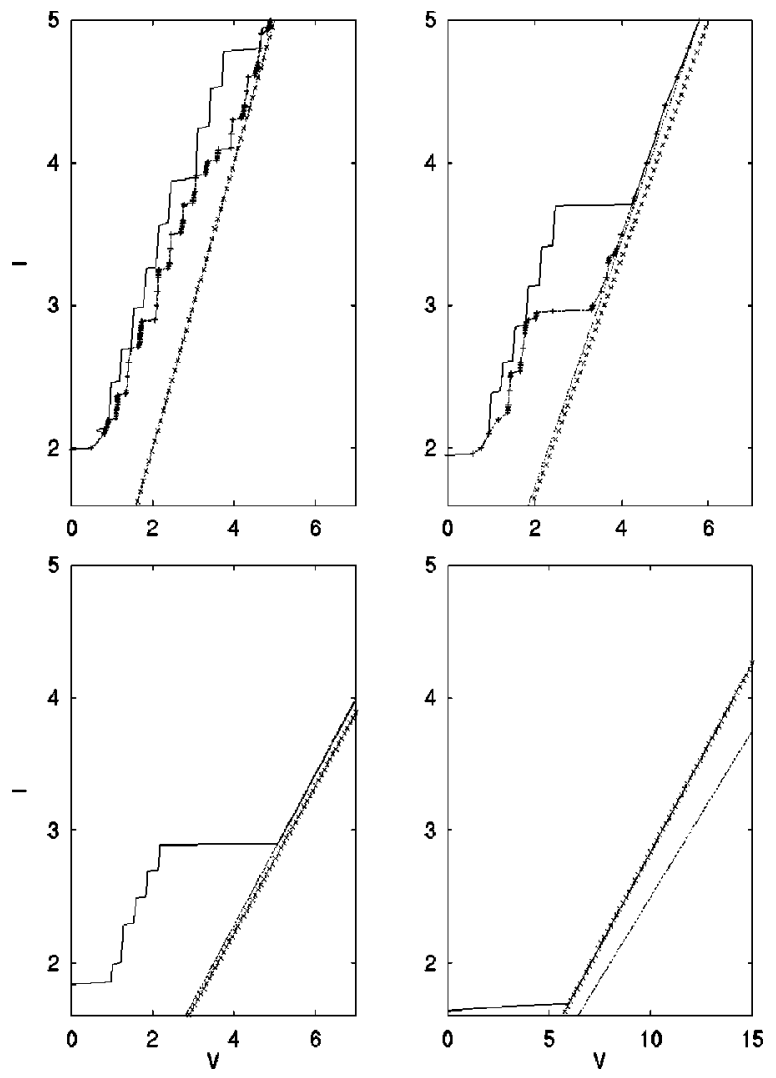

FIG. 8. I-V characteristic $z=2, \lambda / \alpha=0$ (top left), 0.4 (top right), 1.6 (bottom left) and 4 (bottom right). In the top two panels, the points of the $I-V$ curve marked by the $(+)$ sign correspond to increasing current and the plain line to decreasing current. The benchmarks are indicated as: HVL $(* * * * * * * *)$ and LFL $(\cdots \cdots \cdots)$.

completely for $\lambda / \alpha=4$. For this large value of $\lambda$, the HVL expression given by Eq. (3.1) provides a better description than the LFL.

These results show the transition from a state where the system is coupled to a cavity mode and the voltage is fixed to the laminar flow regime for which the voltage (and the frequency) is a linear function of the applied current. To study this transition, we have fixed the current to $I=3$ and plotted in Fig. 9 the traveling wave speed $\left(\varphi_{t} / \varphi_{x}\right)$ for $z=2$ for two different values of $\lambda / \alpha$ : (i) $\lambda / \alpha=0$, indicated by ( $\diamond$ ), and (ii)

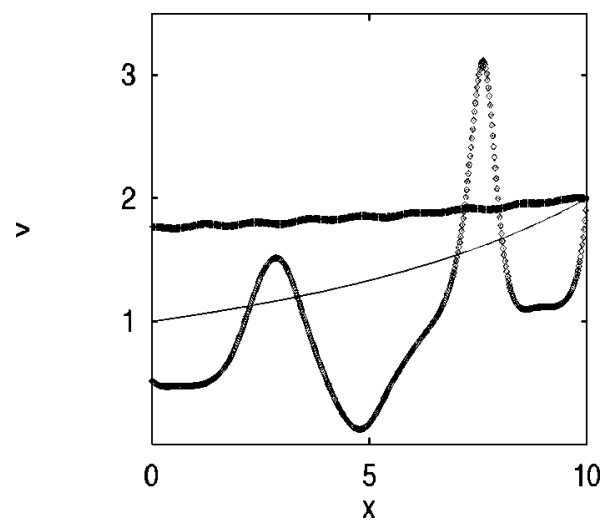

FIG. 9. Plot of the traveling wave speed $-\varphi_{t} / \varphi_{x}$ as a function of $x$ at a particular time for $\lambda / \alpha=0(\diamond)$ and $\lambda / \alpha=1.6(\times), z=2$ and $I=3$. The corresponding LFL approximations are shown as solid lines.
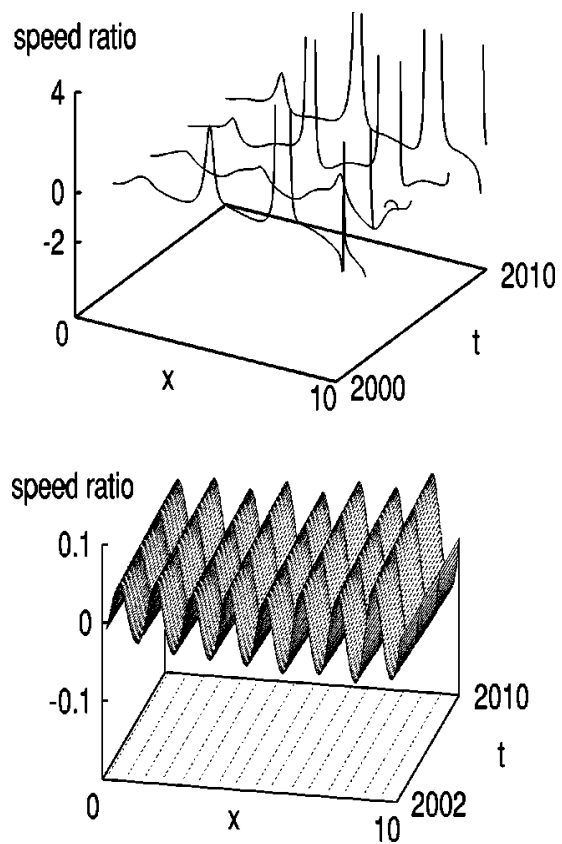

FIG. 10. Plot of the relative traveling wave speed ratio $\left(v-v_{\mathrm{LFL}}\right) / v_{\mathrm{LFL}}$ as a function of $x$ and $t$ for two points in the $I-V$ curve presented in the bottom left panel of Fig. $8(\lambda / \alpha=1.6), I=2.89$ (top panel) and 2.90 (bottom panel). The zero value contour lines are plotted as dashed lines.

$\lambda / \alpha=1.6$, indicated by $(\mathrm{X})$. Also on this figure the corresponding LFL approximations are plotted as solid lines.

Evidently the LFL approximation for laminar flow does not give a good description for $\lambda / \alpha=0$, because there are oscillations of the velocity around unity that are not taken into account. These oscillations correspond to alternating compressions and dilatations of the helical wave, stemming from the mismatched load.

With $\lambda / \alpha=1.6$, on the other hand, the agreement is very good; the numerical calculations and the LFL approximation are practically indistinguishable. Thus the velocity is approximately constant indicating that the fluxon chain is moving smoothly toward the load, and as expected the LFL provides a good description. In other words, Fig. 9 demonstrates the value of exponential tapering of the junction in order to obtain laminar flow.

A big advantage of laminar flow is that the temporal behavior of the solution is periodic leading to a minimal linewidth for the oscillator. This is not necessarily the case in the resonant regime as shown in Fig. 10 where we plot the speed for two values of the current $I$ in the $\mathrm{I}-\mathrm{V}$ characteristic curve for $\lambda / \alpha=1.6$ of Fig. 8, at four successive times. The top panel is for a current $I=2.89$ in the resonant regime while the bottom panel corresponds to $I=2.9$ in the laminar flow regime. The solution for $I=2.89$ is chaotic with large oscillations of $v$ around 1 due to the changing of sign of $\varphi_{x}$. The temporal behavior of the voltage is irregular leading to a large spectral width. ${ }^{7}$ For $I=2.9$ the speed has jumped to about 1.8 and the ratio is very small indicating that the solution follows the laminar flow benchmark. We estimated $A$ to be about 10 and this clearly points out to a type 3 supraluminous helical wave. 

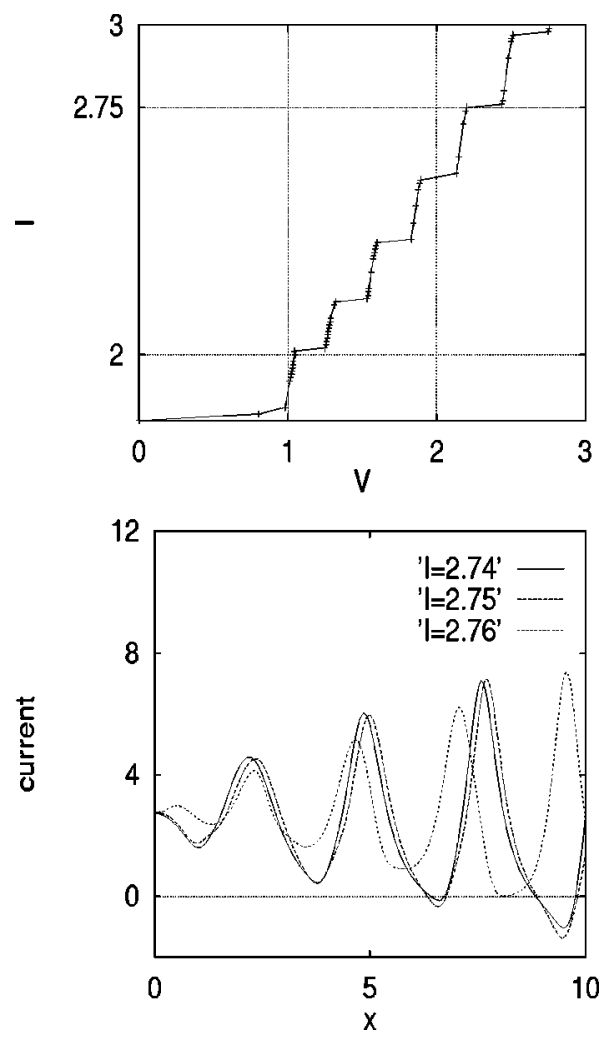

FIG. 11. $I-V$ characteristic (top panel) and corresponding $\varphi_{x}$ (bottom panel). The parameters are $z=1.4$ and $\lambda / \alpha=2$. The plot of $\varphi_{x}$ is given for $I=2.74,2.75$, and 2.76 .

\section{NONLAMINAR FLOW}

It is evident from the results presented above that laminar flow is not to be expected when the input current $I$ is slightly larger than the threshold $I_{\theta}$. In this section we present a variety of numerical data indicating the several ways that laminar flow can be disturbed in a flux flow oscillator and showing that these phenomena can be mitigated through an exponentially tapered design.

\section{A. Nature of the resonances}

Here we present and analyze numerical data related to the resonances (or steps in the $I-V$ characteristics) at smaller values of $I-I_{\theta}$. The theoretical arguments are based on a linear stability analysis of the laminar flow solution for $z$ large that is carried out in Appendix B. There we show that the coefficient $a_{n}$ of the $n$th Fourier mode $\cos (n \pi x / L)$ grows when $V=n \pi / L$. This occurs at current intervals $\Delta I$ given by

$$
\Delta I=\frac{\pi(2+2 \alpha L z-\lambda L)}{(2+\lambda L) L z}
$$

A typical example is shown in Fig. 11, where the characteristic curve is plotted in the top panel and the bottom panel shows $\varphi_{x}$ for three different values of $I$ close to $I$ $=2.75$. The resonance steps occur for $V=n \pi L$ and are related to the number of fluxons in the junction. For $I=2.74$ and $2.75, V \approx 7 \pi / L$, there are three maxima in the plot indicating the presence of three fluxons in the junction, whereas for $I=2.76$ one has stepped to the next branch $V \approx 8 \pi / L$ and the fluxon count is four. Thus the resonance steps are related
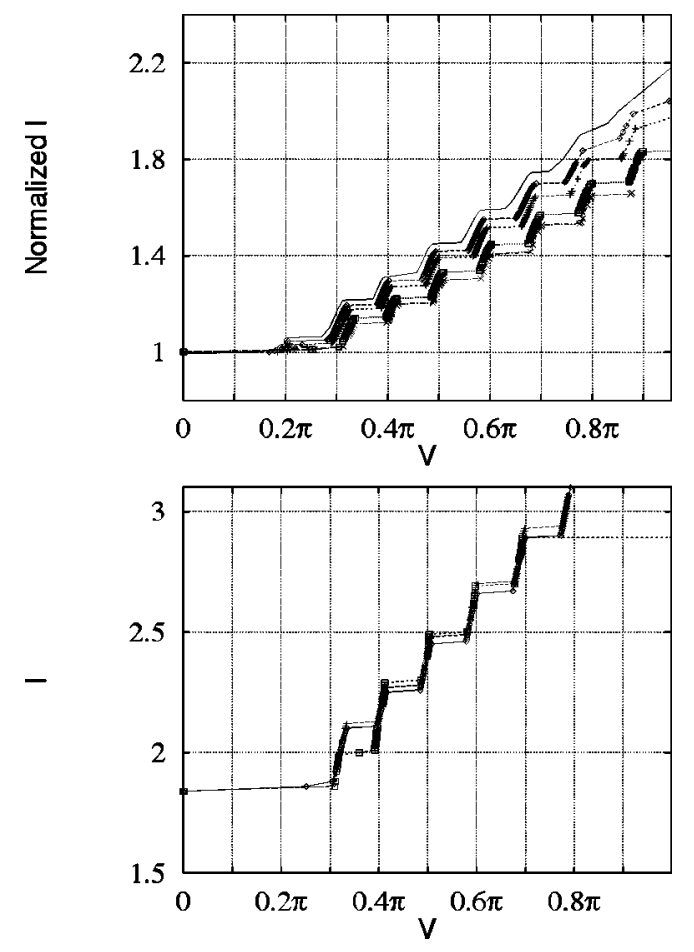

FIG. 12. Top panel: the $I-V$ characteristic curves for $\lambda / \alpha=0$ (top curve), $0.4,0.8,1.6$, and 2 (bottom curve), where the current $I$ has been rescaled by the threshold value $I_{\theta}(z=1.4)$. Bottom panel: the $I-V$ characteristic curves for $\lambda / \alpha=1.6$ and $z=1.4,1.6$, and 2 .

to the number of fluxons on the junction. Notice also that the resonant spacing $\Delta I=0.18$ is in agreement with the four resonances observed between $I=2$ and 2.75 .

The top panel of Fig. 12 presents the $I-V$ characteristic curves for different values of $\lambda$ where the $I$ has been rescaled by the threshold value $I_{\theta}=2(1-\lambda)$, indicating that resonance steps occur for $V_{n}=n \pi / L$. Notice that the steps do not exactly superpose, the voltage increases slightly with $\lambda$ as expected from a linear analysis. To show the effect of changing the load for a fixed $\lambda / \alpha=1.6$, we present in the bottom panel of Fig. 12 the characteristic curves for $z=1.4,1.6$, and 2 , where again the resonances are seen at the values $V_{n}$ $=n \pi / L$. Here $\Delta I=0.20$ which gives five resonances between $I=2$ and $I=3$. In the case of Fig. 8 where $z=2$, the resonance spacing $\Delta I$ is reduced from $\Delta I=0.314$ for $\lambda / \alpha$ $=0$ (about 10 resonances between $I=2$ and 5) to $\Delta I=0.27$ for $\lambda / \alpha=0.4$ (6 resonances between $I=2$ and 3.8) to $\Delta I$ $=0.08$ for $\lambda / \alpha=4$. Therefore when $z$ is large, we observe a strong decrease of the resonance spacing when the tapering is increased. This explains the absence of resonances in the $I-V$ characteristics for the large value of $\lambda / \alpha$ in Figure 8.

Appendix B shows also that resonant steps exist only for $V_{n}$ where $n \leqslant L$. Larger values of $n$ will cause very small values of the forcing coefficients in the evolution equation of the amplitude of the Fourier mode $\cos (n \pi x / L)$. In other words, a junction of length $L=10$ will exhibit about 10 resonant steps, and a junction of length $L=20$ the double. This is confirmed by the simulations. 

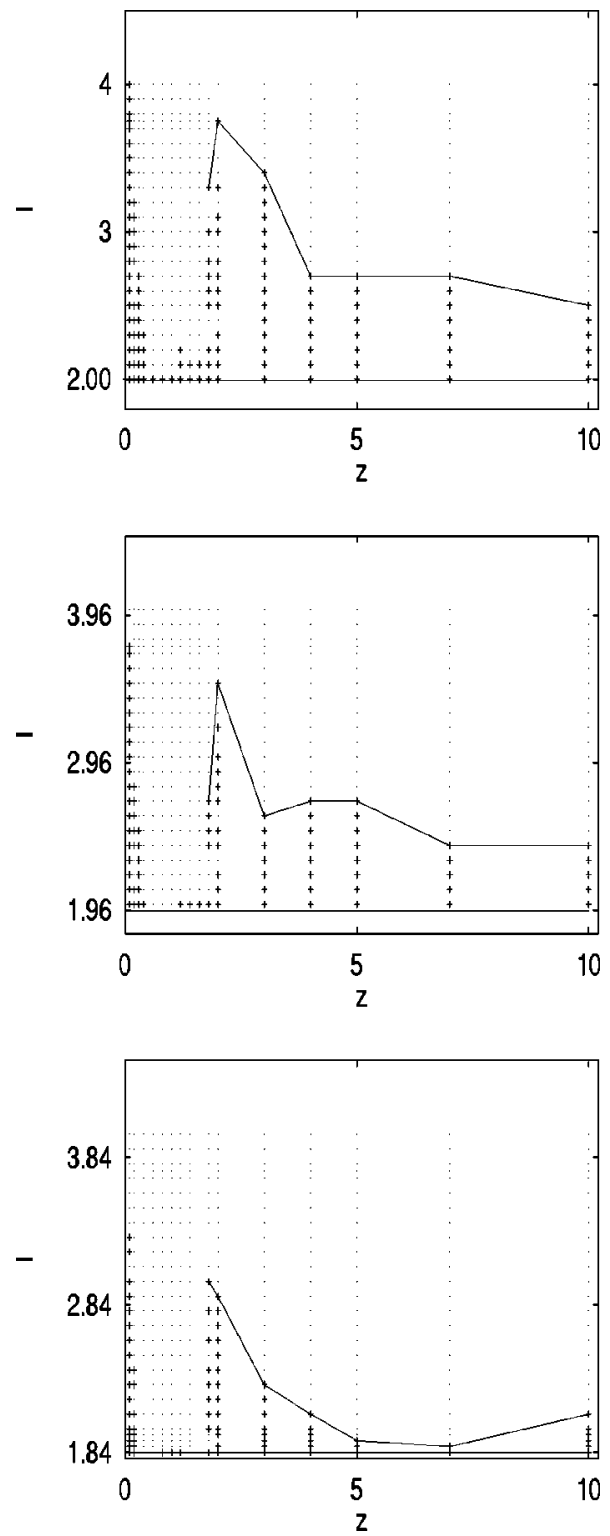

FIG. 13. Laminar flow boundaries in parameter space $(I, z)$ for $\lambda / \alpha=0$ (top), 0.4 (middle), and 1.6 (bottom).

\section{B. $(\lambda, I, z)$ parameter space}

For the reliable design of a flux flow oscillator, it is important to know how the region of laminar flow depends upon the device parameters; thus some relevant information is presented in this section.

Figure 13 shows the regions of laminar and nonlaminar flow in the parameter space $(I, z)$ for $\lambda / \alpha=0$ (top), 0.4 (middle), and 1.6 (bottom). The diamonds (in the lower parts of the figures) indicate nonlaminar flow, while the dots (in the upper parts of the figures) show where laminar flow was observed. The $I$ (current) scale is the same for the three plots. These data demonstrate how the parameter range of laminar flow grows with increasing $\lambda$ (exponential tapering of the junction).

Of primary importance in oscillator design is the power delivered to the load. Some relevant data are shown in the top panel of Fig. 14, where the solid lines indicate the output power calculated under the assumption of laminar flow from
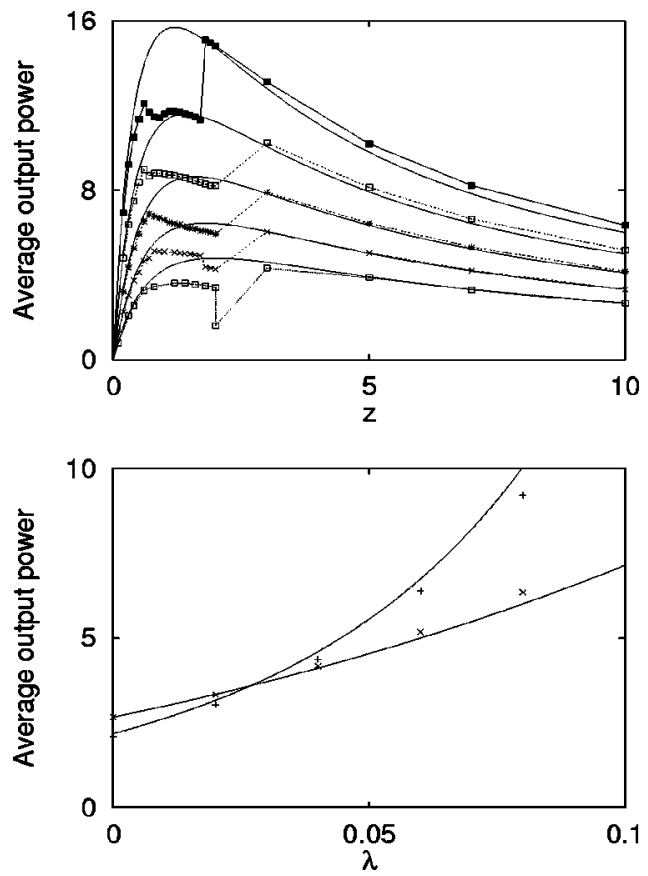

FIG. 14. Average output power in the load vs $z$ (top panel) with $I=3.1$, $\lambda / \alpha=0(\diamond), 0.4(+), 0.8(\square), 1.2(\times), 1.6(\triangle)$. The bottom panel shows the average output power into the load vs $\lambda$ for $I=3.1$ and $z=0.3$ $(\diamond)$ and $10(+)$. In both panels the solid lines represent laminar flow solutions.

Eq. (3.5). For both small and large values of $z$, the numerical results agree rather well with the idealized assumption of laminar flow, and as expected from Fig. 13 this agreement improves with increased tapering $(\lambda)$ of the junction.

In the range $0.5<z<2$ the average output power does not follow the benchmarks for the value of the current considered. This is the region where the speed of the helical wave is around 1 so that instabilities and resonances occur. Larger values of $z$ lead to less resonances and the numerical solution follows the benchmarks as shown in the I-V characteristics of Fig. 8.

The bottom panel of Fig. 14 shows how the output power into a small load $(z=0.3)$ and a large load $(z=10)$ increases with junction tapering $(\lambda)$, clearly demonstrating the design advantage of a tapered structure.

\section{Regularization of standard flux flow}

Often a flux flow oscillator is designed with an external magnetic field and transverse bias current. To study the effect of exponentially tapering the width of such a junction, we introduce a transverse bias current $(\gamma)$ by augmenting Eq. (2.4) to

$$
\frac{\partial^{2} \varphi}{\partial t^{2}}-\frac{\partial^{2} \varphi}{\partial x^{2}}+\sin \varphi+\lambda \frac{\partial \varphi}{\partial x}+\alpha \frac{\partial \varphi}{\partial t}-\gamma=0,
$$

and including a magnetic field $\eta$ in the boundary conditions (2.2). In a standard overlap junction geometry, the transverse bias current is uniform in the $x$ direction, forcing fluxons toward the load. ${ }^{4}$

From the results presented above, we expect that for $\lambda / \alpha=0.8$ the system will follow the benchmarks and exhibit 

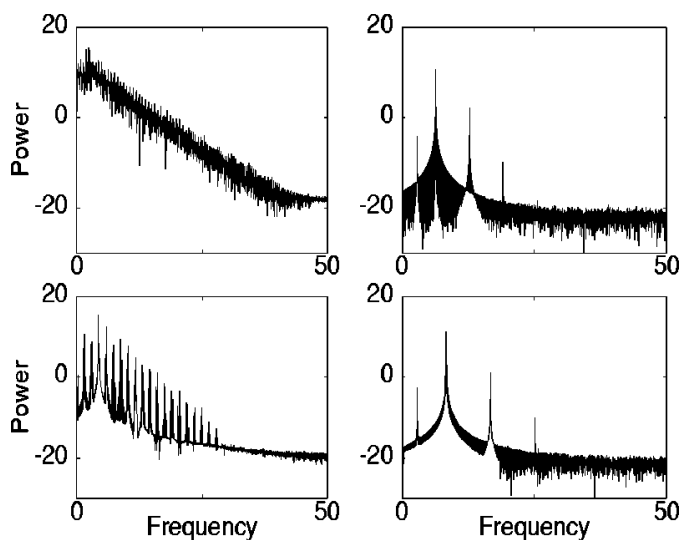

FIG. 15. Fourier spectrum of the voltage output in the case of a standard flux flow oscillator with external magnetic field $\eta=2$ and transverse bias currents $\gamma=0.2$ (top) and 0.3 (bottom), and for $\lambda / \alpha=0$ (left) and 0.8 (right). The load is $z=10^{4}$.

a periodic temporal behavior. The spectral purity of the voltage should therefore be minimal in the tapered case. In Fig. 15 are plotted Fourier spectra of the output voltage for $\eta$ $=2$ and two levels of transverse bias $(\gamma=0.2$ and 0.3$)$ for no tapering $(\lambda / \alpha=0)$ on the left hand side of the figure, and for modest tapering $(\lambda / \alpha=0.8)$ on the right hand side of the figure. In these computations, the load is $z=10^{4}$. The spectra for the tapered device (on the right) are associated to a periodic signal while the ones for the untapered system (on the left) indicate a chaotic voltage. As expected, the spectral purity of the flux flow oscillator is substantially improved by introducing an exponential taper into the design.

\section{Influence of the surface damping}

Finally we briefly consider the influence of damping of a flux flow oscillator caused by surface currents (parallel to the insulating barrier of the junction), ${ }^{11}$ which has hitherto been neglected in our analysis of Eq. (2.1) by assuming that the parameter $\beta$ was zero. (Some numerical details related to these computations are presented in Appendix D.)

To this end, we have compared three $I-V$ characteristics with $\lambda / \alpha=0.4$ : (i) $\beta=0$, (ii) 0.01 , and (iii) 0.05 . Our calculations at these levels of surface damping have little influence on the behavior of the oscillator, indicating that analyses neglecting surface damping can be expected to yield reliable results.

\section{CONCLUSIONS}

The broad conclusion to be drawn from the numerical and analytic studies presented here is that exponential tapering of a Josephson flux flow oscillator substantially improves its performance. In particular:

(1) Tapering the junction enlarges the region of parameter space over which laminar flow is observed.

(2) Tapering substantially increases the power output of the oscillator.

(3) Tapering substantially increases the spectral purity of the oscillator.
As can be seen from Fig. 15, the increase of spectral purity with exponential tapering is significant for a standard flux flow oscillator in an external magnetic field and transverse bias current. Since exponential tapering is not expected to increase the cost or difficulty of fabrication of a flux flow oscillator, we suggest that this feature should be incorporated in future designs.

\section{ACKNOWLEDGMENTS}

The authors thank N. F. Pedersen, V. Koshelets, and A. V. Ustinov for helpful discussions. We are pleased to acknowledge the support of European Network grant CHRXCT93-0331. Also A. B. and J. G. C. thank the Department of Mathematical Modelling at the Technical University of Denmark for hospitality during several visits when this work was completed. A. B. is grateful to the Laboratoire de Physique Mathématique de l'Université de Montpellier II for its hospitality during several visits. The authors also thank the CRIHAN computing center in Rouen for the use of its facilities.

\section{APPENDIX A: DERIVATION OF THE LINEAR FLOW LIMIT (LFL)}

The variational analysis leading to the linear flow approximation to laminar flow is based on the conservation of the energy of the system that is given by the sine-Gordon Hamiltonian $^{4}$

$$
H=\int_{0}^{L}\left[\frac{1}{2}\left(\frac{\partial \varphi^{2}}{\partial t}+\frac{\partial \varphi^{2}}{\partial x}\right)+(1-\cos \varphi)\right] d x,
$$

from which it can be shown that $H$ satisfies the power balance equation ${ }^{4,7}$

$$
\begin{aligned}
\frac{d H}{d t}= & I \frac{\partial \varphi}{\partial t}(0, t)-z\left(\frac{\partial \varphi}{\partial x}\right)^{2}(L, t)-\alpha \int_{0}^{L}\left(\frac{\partial \varphi}{\partial t}\right)^{2} d x \\
& -\lambda \int_{0}^{L} \frac{\partial \varphi}{\partial x} \frac{\partial \varphi}{\partial t} d x .
\end{aligned}
$$

In the stationary regime the average $d H / d t=0$, so

$$
\begin{gathered}
I\left\langle\frac{\partial \varphi}{\partial t}(0, t)\right\rangle-z\left\langle\left(\frac{\partial \varphi}{\partial x}\right)^{2}(L, t)\right\rangle-\alpha\left\langle\int_{0}^{L}\left(\frac{\partial \varphi}{\partial t}\right)^{2} d x\right\rangle \\
-\lambda\left\langle\int_{0}^{L} \frac{\partial \varphi}{\partial x} \frac{\partial \varphi}{\partial t} d x\right\rangle=0 .
\end{gathered}
$$

The voltage is given by the formula

$$
V \equiv\left\langle\frac{\partial \varphi}{\partial t}\right\rangle=\frac{1}{T} \int_{0}^{T} \frac{\partial \varphi}{\partial t}(x, t) d t .
$$

For sufficiently large $I$

$$
\left\langle\left(\frac{\partial \varphi}{\partial t}\right)^{2}\right\rangle=\left\langle\frac{\partial \varphi}{\partial t}\right\rangle^{2}
$$

thus, using the right boundary conditions we have

$$
\left\langle z\left(\frac{\partial \varphi}{\partial x}\right)^{2}(L, t)\right\rangle=\frac{1}{z}\left\langle\left(\frac{\partial \varphi}{\partial t}\right)^{2}(L, t)\right\rangle=\frac{1}{z} V^{2}
$$


and

$$
\alpha\left\langle\int_{0}^{L}\left(\frac{\partial \varphi}{\partial t}\right)^{2} d x\right\rangle=\alpha L V^{2} .
$$

In Eq. (A3) we need the quantity

$$
\left\langle\int_{0}^{L} \frac{\partial \varphi}{\partial x} \frac{\partial \varphi}{\partial t} d x\right\rangle
$$

and to obtain this, we interpolate linearly the current $\partial \varphi / \partial x$ using the information given by the boundary conditions at $x=0$ and $L$, thus

$$
\frac{\partial \varphi}{\partial x}(x, t) \doteq \frac{I z+\varphi_{t}(L, t)}{z L} x-I .
$$

Substituting this into Eq. (A4) gives

$$
\begin{aligned}
& \left\langle\int_{0}^{L} \frac{\partial \varphi}{\partial x} \frac{\partial \varphi}{\partial t} d x\right\rangle \\
& \quad=\left\langle\int_{0}^{L}\left(-I+\frac{I}{L} x\right) \frac{\partial \varphi}{\partial t} d x\right\rangle+\left\langle\int_{0}^{L} x \frac{\varphi_{t}(L, t)}{z L} \frac{\partial \varphi}{\partial t} d x\right\rangle,
\end{aligned}
$$

and using Fubini's theorem ${ }^{15}$

$$
\begin{aligned}
& \left\langle\int_{0}^{L}\left(-I+\frac{I}{L} x\right) \frac{\partial \varphi}{\partial t} d x\right\rangle \\
& \quad=\frac{1}{T} \int_{0}^{T} \int_{0}^{L}\left(-I+\frac{I}{L} x\right) \frac{\partial \varphi}{\partial t} d x d t \\
& =\int_{0}^{L}\left(-I+\frac{I}{L} x\right) \frac{1}{T} \int_{0}^{T} \frac{\partial \varphi}{\partial t} d t d x=-\frac{I L}{2} V,
\end{aligned}
$$

and

$$
\begin{aligned}
& \left\langle\int_{0}^{L} \frac{x}{z L} \frac{\partial \varphi}{\partial t}(L, t) \frac{\partial \varphi}{\partial t}(x, t) d x\right\rangle \\
& \quad=\frac{1}{T} \int_{0}^{T} \int_{0}^{L} \frac{x}{z L} \frac{\partial \varphi}{\partial t}(L, t) \frac{\partial \varphi}{\partial t}(x, t) d x d t \\
& =\int_{0}^{L} \frac{x}{z L} \int_{0}^{T} \frac{\partial \varphi}{\partial t}(L, t) \frac{\partial \varphi}{\partial t}(x, t) d t d x=\frac{L}{2 z} V^{2} .
\end{aligned}
$$

Thus

$$
\lambda\left\langle\int_{0}^{L} \frac{\partial \varphi}{\partial x} \frac{\partial \varphi}{\partial t} d x\right\rangle=-\frac{\lambda L}{2}\left(\frac{V}{z}+I\right) V .
$$

Regrouping all these terms we obtain the voltage as

$$
V \doteq \frac{(2+\lambda L) z I}{2+2 \alpha L z-\lambda L} \text {. }
$$

\section{APPENDIX B: LINEAR STABILITY ANALYSIS OF THE HVL SOLUTION AND RESONANCES}

Let us write the solution of Eq. (2.4) as $\varphi=\varphi_{0}+\psi$ where $\varphi_{0}$ is the HVL solution and $|\psi| \ll 1$. Then $\psi$ satisfies

$$
\psi_{t t}-\psi_{x x}+\sin \varphi_{0}+\cos \varphi_{0} \psi=-\alpha \psi_{t}-\lambda \psi_{x},
$$

together with the boundary conditions

$$
\left.\psi_{x}\right|_{x=0}=0,\left.\quad \psi_{x}\right|_{x=L}=0,
$$

where we choose to neglect $1 / z$.

It is then natural to decompose $\psi$ in a cosine Fourier series

$$
\psi(x, t)=\sum_{n} a_{n}(t) \cos \frac{n \pi x}{L} .
$$

By substituting this into Eq. (B1) and projecting on $\cos p \pi x / L$ we get

$$
\begin{aligned}
\ddot{a}_{p}+ & a_{p}\left(\frac{p \pi}{L}\right)^{2}+\frac{2}{L} \int_{0}^{L} \sin \varphi_{0} \cos \frac{p \pi x}{L} d x \\
& +\sum_{n} a_{n} \frac{2}{L} \int_{0}^{L} \cos \varphi_{0} \cos \frac{p \pi x}{L} \cos \frac{n \pi x}{L} d x \\
& =-\alpha \dot{a}_{p}+\lambda \sum_{n} a_{n} \frac{n \pi}{L} \frac{2}{L} \int_{0}^{L} \sin \frac{n \pi x}{L} \cos \frac{p \pi x}{L} d x .
\end{aligned}
$$

It can be seen that the last term of the above expression is zero for all values of $n$ and $p$.

We then separate the temporal part of $\varphi_{0}$ by writing $\varphi_{0}(x, t)=V t+f_{0}(x)$. This gives the following evolution for $a_{p}$.

$$
\begin{gathered}
\ddot{a}_{p}+a_{p}\left(\frac{p \pi}{L}\right)^{2}\left[1+C_{p} \sin V t+D_{p} \cos V t\right] \\
+A_{p} \sin V t+B_{p} \cos V t=-\alpha \dot{a}_{p},
\end{gathered}
$$

where the other terms for $n \neq p$ have been neglected and the coefficients $A_{p}, B_{p}, C_{p}$, and $D_{p}$ are given by

$$
\begin{aligned}
& A_{p}=\frac{2}{L} \int_{0}^{L} \cos f_{0} \cos \frac{p \pi x}{L} d x, \\
& B_{p}=\frac{2}{L} \int_{0}^{L} \sin f_{0} \cos \frac{p \pi x}{L} d x, \\
& C_{p}=-\frac{2 L}{(\pi p)^{2}} \int_{0}^{L} \sin f_{0} \cos ^{2} \frac{p \pi x}{L} d x, \\
& D_{p}=\frac{2 L}{(\pi p)^{2}} \int_{0}^{L} \cos f_{0} \cos ^{2} \frac{p \pi x}{L} d x .
\end{aligned}
$$

This evolution of $a_{p}$ leads to linear or parametric resonances when $V=p \pi / L$ depending on the coefficients. To estimate them, one can approximate $f_{0}$ by writing it as $f_{0}(x)=\theta x / L$ where $\theta$ is the fluxon content in the junction.

When $\theta=p \pi, A_{p}=1, B_{p}=0, C_{p}=0$, and $D_{p}=(1$ $\left.-(-1)^{p}\right) L^{2} /(p \pi)^{3}$. For $\theta=p \pi+\pi / 2, B_{p}$ is maximum. This shows that if $V=p \pi / L$ one excites linearly the mode $\cos p \pi x / L$ and parametrically the mode $\cos p \pi x / 2 L$. This is possible as long as the factor $p \pi / L$ remains of order 1 . This is the case shown in the bottom panel of Fig. 12 when $V$ $=7 \pi / L$. The mode $\cos 3 \pi x / L$ is excited parametrically because $A_{3}=B_{3}=C_{3}=0$ and $D_{3} \approx 0.25$.

The values of $I$ for which there is a resonance can be obtained by identifying the voltage for the HVL model (3.3) with $p \pi / L$ giving equidistant values for the current separated by 


$$
\Delta I=\frac{\pi(2+2 \alpha L z-\lambda L)}{(2+\lambda L) z L} .
$$

\section{APPENDIX C: THE MATCHED LOAD CONDITION IN THE PRESENCE OF SURFACE DAMPING}

The modified sine-Gordon equation with $\beta$-term reads ${ }^{7}$

$$
\frac{\partial^{2} \varphi}{\partial x^{2}}-\frac{\partial^{2} \varphi}{\partial t^{2}}-\sin \varphi=\alpha \frac{\partial \varphi}{\partial t}+\lambda \frac{\partial \varphi}{\partial x}+\lambda \beta \frac{\partial^{2} \varphi}{\partial x \partial t}-\beta \frac{\partial^{3} \varphi}{\partial x^{2} \partial t},
$$

where the left boundary conditions of $(\mathrm{C} 1)$ can be obtained as

$$
\frac{\partial \varphi}{\partial x}+\left.\beta \frac{\partial^{2} \varphi}{\partial x \partial t}\right|_{x=0}=-I
$$

The right boundary condition is an ohmic equation $V=z I$, which in terms of $\varphi$ was written as

$$
-\left.\frac{\partial \varphi}{\partial t}\right|_{x=L}=\left.z \frac{\partial \varphi}{\partial x}\right|_{x=L}
$$

Including the $\beta$ term the current is not $\partial \varphi / \partial x$ but

$$
\left(1+\beta \frac{\partial}{\partial t}\right) \frac{\partial \varphi}{\partial x}
$$

as above, so the new boundary condition becomes

$$
\frac{\partial \varphi}{\partial x}+\left.\beta \frac{\partial^{2} \varphi}{\partial x \partial t}\right|_{x=L}=-\left.\frac{1}{z} \frac{\partial \varphi}{\partial t}\right|_{x=L} .
$$

Assuming a single fluxon solution of Eq. (C1), and a long junction for which

$$
\varphi(x, t)=4 \arctan \left(\exp \left(\frac{x-v t}{\sqrt{1-v^{2}}}\right)\right)
$$

and

$$
\frac{\partial \varphi}{\partial x}=\frac{2}{\sqrt{1-v^{2}}} \operatorname{sech}\left(\frac{x-v t}{\sqrt{1-v^{2}}}\right)
$$

we have the following ordinary differential equation for $v$ :

$$
\dot{v}=\left(1-v^{2}\right)(\lambda-\alpha v)-\beta \frac{v}{3},
$$

where a fixed point for $(\mathrm{C} 4)$ satisfies

$$
\alpha v^{3}-\lambda v^{2}-\left(\alpha+\frac{\beta}{3}\right) v+\lambda=0 .
$$

Supposing that $v_{0}=\lambda / \alpha$ is the solution when $\beta=0$, we can write

$$
v=v_{0}+\beta v_{1}+O\left(\beta^{2}\right) .
$$

Substituting

$$
\begin{gathered}
\alpha\left(v_{0}^{3}+3 \beta v_{0} v_{1}\right)-\lambda\left(v_{0}^{2}+2 \beta v_{0} v_{1}\right) \\
-\left(v_{0}-\beta v_{1}\right)\left(\alpha+\frac{\beta}{3}\right)+\lambda=0,
\end{gathered}
$$

gives at

$$
\begin{aligned}
& O\left(\beta^{0}\right) \quad \alpha v_{0}^{3}-\lambda v_{0}^{2}-\alpha v_{0}+\lambda=0, \\
& O\left(\beta^{1}\right) \quad 3 \alpha v_{0}^{2} v_{1}-2 \lambda v_{0} v_{1}-\frac{v_{0}}{3}-\alpha v_{1}=0,
\end{aligned}
$$

implying

$$
v_{1}=\frac{\lambda}{3\left(\lambda^{2}-\alpha^{2}\right)} .
$$

Then the condition of matched load impedance is

$$
v=\frac{\lambda}{\alpha}+\frac{\lambda \beta}{3\left(\lambda^{2}-\alpha^{2}\right)} .
$$

To obtain the output power, multiply Eq. (C1) by $\partial \varphi / \partial t$ and integrate between 0 and $L$ :

$\frac{d H}{d t}=-\alpha \int_{0}^{L}\left(\frac{\partial \varphi}{\partial t}\right)^{2} d x-\lambda \int_{0}^{L} \frac{\partial \varphi}{\partial x} \frac{\partial \varphi}{\partial t} d x-\beta \int_{0}^{L}\left(\frac{\partial^{2} \varphi}{\partial x \partial t}\right)^{2} d x$

$$
+\left[\frac{\partial \varphi}{\partial x} \frac{\partial \varphi}{\partial t}\right]_{0}^{L}+\beta\left[\frac{\partial \varphi}{\partial t} \frac{\partial^{2} \varphi}{\partial x \partial t}\right]_{0}^{L}-\frac{\lambda \beta}{2}\left[\left(\frac{\partial \varphi}{\partial t}\right)^{2}\right]_{0}^{L}
$$

where $H$ is the sine-Gordon Hamiltonian

$$
H=\int_{0}^{L}\left[\frac{1}{2}\left(\frac{\partial \varphi^{2}}{\partial t}+\frac{\partial \varphi^{2}}{\partial x}\right)+(1-\cos \varphi)\right] d x .
$$

Using the boundary conditions from Eqs. (C2) and (C3), we obtain

$\frac{d H}{d t}=-\alpha \int_{0}^{L}\left(\frac{\partial \varphi}{\partial t}\right)^{2} d x-\lambda \int_{0}^{L} \frac{\partial \varphi}{\partial x} \frac{\partial \varphi}{\partial t} d x-\beta \int_{0}^{L}\left(\frac{\partial^{2} \varphi}{\partial x \partial t}\right)^{2} d x$

$$
\begin{aligned}
& -\left(\frac{\partial \varphi}{\partial t}\right)^{2}(L, t)\left(\frac{1}{z}+\frac{\lambda \beta}{2}\right) \\
& -\frac{\partial \varphi}{\partial t}(0, t)\left(-I-\frac{\lambda \beta}{2} \frac{\partial \varphi}{\partial t}(0, t)\right) .
\end{aligned}
$$

Thus the output power into the load is

$$
P(t)=\left(\frac{\partial \varphi}{\partial t}(L, t)\right)^{2}\left(\frac{1}{z}+\frac{\lambda \beta}{2}\right) .
$$

\section{APPENDIX D: THE NUMERICAL SCHEME IN THE PRESENCE OF SURFACE DAMPING}

To numerically integrate Eq. (C1), we have transformed it into the system of partial differential equations

$$
\left\{\begin{array}{l}
\varphi=\psi \\
\psi_{t}=\varphi_{x x}-\sin (\varphi)-\alpha \psi-\lambda \varphi-\lambda \beta \psi_{x}+\beta \psi_{x x},
\end{array}\right.
$$

with

$$
\left.\left(\varphi_{x}+\beta \psi_{x}\right)\right|_{x=0}=-I ;\left.\quad\left(z \varphi_{x}+z \beta \psi_{x}\right)\right|_{x=L}=-\left.\psi\right|_{x=L} .
$$

This equation has been solved using a method of lines, ${ }^{12}$ where the time operator is advanced using an ordinary differential equation solver and the right hand sides is discretized using finite differences 
$\left\{\begin{array}{l}\dot{\varphi}_{n}=\psi_{n} \\ \dot{\psi}_{n}=f\left(\varphi_{n-1}, \varphi_{n}, \varphi_{n+1}, \psi_{n-1}, \psi_{n}, \psi_{n+1}\right) \text { for } n=0,1, . ., N .\end{array}\right.$

At the ends, $n=0, N$, this system needs two conditions in space for $\varphi\left(\varphi_{-1}, \varphi_{N+1}\right)$ and two for $\psi\left(\psi_{-1}, \psi_{N+1}\right)$.

For $x=0$, we have

$$
\left\{\begin{array}{l}
\varphi_{x}+\beta \psi_{x}=-I \\
\psi_{x}+\beta \psi_{x t}=0
\end{array}\right.
$$

Substituting the operator $\psi_{t}$ from Eq. (D1) in the second part of Eq. (D2), we obtain

$$
\left\{\begin{array}{l}
\varphi_{x}+\beta \psi_{x}=-I \\
\psi_{x}(1-\alpha \beta)-\lambda \beta^{2} \psi_{x x}+\beta^{2} \psi_{x x x} \\
\quad-\beta \cos (\varphi) \varphi_{x}-\lambda \beta \varphi_{x x}+\beta \varphi_{x x x}=0 .
\end{array}\right.
$$

The first part of Eq. (D2) can be discretized to give

$$
\varphi_{-1}+\beta \psi_{-1}=\varphi_{1}+\beta \psi_{1}+2 h I .
$$

The trick is now to use in the second part of Eq. (D2) expressions for $\psi_{x x x}$ and $\varphi_{x x x}$ that involve $\psi_{-1}^{k}$ and $\varphi_{-1}^{k}$ and not more points on the left. To this end, we approximate $\psi_{x x x}$ at $x=0$ using five points neighborhood ${ }^{16}$

$$
\begin{aligned}
\left.\psi_{x x x}\right|_{0}= & \frac{1}{h^{3}}\left(-\frac{3}{2} \psi_{-1}+5 \psi_{0}-6 \psi_{1}+3 \psi_{2}-\frac{1}{2} \psi_{3}\right) \\
& +O\left(h^{2}\right) .
\end{aligned}
$$

It can easily be checked that this formula is exact for polynomials of degree $\leqslant 4$.

The second part of Eq. (D2) can then be approximated by

$$
\begin{aligned}
& \frac{\psi_{1}-\psi_{-1}}{2 h}(1-\alpha \beta)-\frac{\psi_{-1}+\psi_{1}-2 \psi_{0}}{h^{2}} \lambda \beta^{2} \\
& +\left(-\frac{3}{2} \psi_{-1}+5 \psi_{0}-6 \psi_{1}+3 \psi_{2}-\frac{\psi_{3}}{2}\right) \frac{\beta^{2}}{h^{3}} \\
& -\beta \cos \left(\varphi_{0}\right) \frac{\varphi_{1}-\varphi_{-1}}{2 h}-\frac{\varphi_{-1}+\varphi_{1}-2 \varphi_{0}}{h^{2}} \lambda \beta \\
& +\left(-\frac{3}{2} \varphi_{-1}+5 \varphi_{0}-6 \varphi_{1}+3 \varphi_{2}-\frac{\varphi_{3}}{2}\right) \frac{\beta}{h^{3}}=0 .
\end{aligned}
$$

Thus the system of Eq. (D3) can be written as

$$
\left\{\begin{array}{l}
\varphi_{-1}+\beta \psi_{-1}=A_{1} \\
B_{1} \varphi_{-1}+C_{1} \psi_{-1}=D_{1}
\end{array}\right.
$$

to yield

$$
\psi_{-1}=\frac{A_{1} B_{1}-D_{1}}{\beta B_{1}-C_{1}} ; \quad \varphi_{-1}=A_{1}-\beta \psi_{-1} .
$$

The same strategy can be applied to the right boundary conditions at $x=L$, where we have

$$
\left\{\begin{array}{l}
\varphi_{x}+\beta \psi_{x}=-\psi / z \\
\psi_{x}+\beta \psi_{x t}+\psi_{t} / z=0 .
\end{array}\right.
$$

Substituting the operator $\psi_{t}=\ldots$ in the second part of Eq. (D5), we obtain

$$
\left\{\begin{array}{l}
\varphi_{x}+\beta \psi_{x}=-\frac{\psi}{z} \\
\left(1-\alpha \beta-\frac{\lambda \beta}{z}\right) \psi_{x}-\left[\beta \cos (\varphi)+\frac{\lambda}{z}\right] \varphi_{x} \\
-\frac{\alpha}{z} \psi-\left(\frac{\beta}{z}+\lambda \beta^{2}\right) \psi_{x x}+\beta^{2} \psi_{x x x}-\frac{1}{z} \sin (\varphi) \\
-\left(\lambda \beta+\frac{1}{z}\right) \varphi_{x x}+\beta \varphi_{x x x}=0 .
\end{array}\right.
$$

We approximate $\psi_{x x x}$ at $x=L$ using five points $\left(x_{N+1}, x_{N}, x_{N-1}, x_{N-2}, x_{N-3}\right)$ neighborhood

$$
\begin{aligned}
\left.\psi_{x x x}\right|_{N}= & \frac{1}{h^{3}}\left(\frac{3}{2} \psi_{N+1}-5 \psi_{N}+6 \psi_{N-1}-3 \psi_{N-2}+\frac{1}{2} \psi_{N-3}\right) \\
& +O\left(h^{2}\right) .
\end{aligned}
$$

It can easily be checked that this formula is exact for polynomials of degree $\leqslant 4$. Thus the first part of Eq. (D5) gives

$$
\varphi_{N+1}+\beta \psi_{N+1}=\varphi_{N-1}+\beta \psi_{N-1}-2 \frac{h}{z} \psi_{N},
$$

and the second equation gives

$$
\begin{aligned}
{\left[-\frac{3}{2} \frac{\beta}{h^{2}}+\frac{1}{2}\left(\beta \cos \left(\varphi_{0}\right)+\frac{\lambda}{z}\right)+\frac{1}{h}\left(\lambda \beta+\frac{1}{z}\right)\right] \varphi_{N+1}+\left[-\frac{3}{2} \frac{\beta^{2}}{h^{2}}+\frac{1}{2}\left(\alpha \beta+\frac{\lambda \beta}{z}-1\right)+\frac{\beta}{h}\left(\lambda \beta+\frac{1}{z}\right)\right] \psi_{N+1} } \\
=-\left[\frac{\alpha}{z} h-\frac{2}{h}\left(\lambda \beta^{2}+\frac{\beta}{z}\right)-5 \frac{\beta^{2}}{h^{2}}\right] \psi_{N}-\left[\frac{1}{2}\left(1-\alpha \beta-\frac{\lambda \beta}{z}\right)+\frac{\beta}{h}\left(\lambda \beta+\frac{1}{z}\right)-6 \frac{\beta^{2}}{h^{2}}\right] \psi_{N-1}+\left[-3 \psi_{N-2}+\frac{1}{2} \psi_{N-3}\right] \frac{\beta^{2}}{h^{2}} \\
\quad-\frac{h}{z} \sin \left(\varphi_{N}\right)+\left[\frac{2}{h}\left(-\lambda \beta+\frac{1}{z}\right)-5 \frac{\beta}{h^{2}}\right] \varphi_{N}-\left[\frac{1}{2}\left(-\beta \cos \left(\varphi_{N}\right)-\frac{\lambda}{z}\right)-\frac{1}{h}\left(-\lambda \beta+\frac{1}{z}\right)-6 \frac{\beta}{h^{2}}\right] \varphi_{N-1} \\
+\left[-3 \varphi_{N-2}+\frac{1}{2} \varphi_{N-3}\right] \frac{\beta}{h^{2}}
\end{aligned}
$$


which can be written as

$$
\left\{\begin{array}{l}
\varphi_{N+1}+\beta \psi_{N+1}=A_{2} \\
B_{2} \varphi_{N+1}+C_{2} \psi_{N+1}=D_{2}
\end{array}\right.
$$

to yield

$$
\psi_{N+1}=\frac{A_{2} B_{2}-D_{2}}{\beta B_{2}-C_{2}} ; \quad \varphi_{N+1}=A_{2}-\beta \psi_{N+1} .
$$

${ }^{1}$ A. V. Ustinov, H. Kohlstedt, and P. Henne, Phys. Rev. Lett. 77, 3617 (1996).

${ }^{2}$ V. P. Koshelets, S. V. Shitov, A. V. Shchukin, L. V. Filippenko, J. Mygind, and A. V. Ustinov, Phys. Rev. B 56, 5572 (1997).

${ }^{3}$ A. C. Scott, Active and Nonlinear Wave Propagation in Electronics (Wiley, New York, 1970).

${ }^{4}$ A. C. Scott, Nonlinear Science: Emergence and Dynamics of Coherent Structures (Oxford University Press, Oxford, 1999).

${ }^{5}$ A. Barone and G. Paterno, Physics and Applications of the Josephson Effect (Wiley, New York, 1982).

${ }^{6}$ G. B. Whitham, Linear and Nonlinear Waves (Wiley, New York, 1974).

${ }^{7}$ A. Benabdallah, J. G. Caputo, and A. C. Scott, Phys. Rev. B 54, 16139 (1996).
${ }^{8}$ A. G. Lindgren and R. J. Buratti, IEEE Trans. Circuit Theory 16, 274 (1969)

${ }^{9}$ E. N. Pelinovskii and S. X. Shavratzkii, Zh. Prikl. Mekhanikii i Tekkincheskoi Fisiki 5, 55 (1974).

${ }^{10} \mathrm{~S}$. Pagano et al., in Nonlinear Superconducting Devices and High $T_{c} \mathrm{Ma}-$ terials, edited by R. D. Parmentier and N. F. Pedersen (World Scientific, Singapore, 1995).

${ }^{11}$ A. C. Scott, Solid-State Electron. 7, 137 (1964).

${ }^{12}$ W. E. Schiesser, The Numerical Method of Lines: Integration of Partial Differential Equations (Academic, New York, 1991).

${ }^{13}$ A. C. Scott, F. Y. F. Chu, and S. A. Reible, J. Appl. Phys. 47, 3272 (1976).

${ }^{14}$ Handbook of Mathematical Functions, edited by M. Abramowitz and I. A. Stegun (Dover, New York, 1972).

${ }^{15}$ W. Rudin, Real and Complex Analysis (McGraw-Hill, New York, 1966).

${ }^{16} \mathrm{~B}$. Demidovitch and I. Maron, Elements de Calcul Numérique (Mir, Moscow, 1987).

${ }^{17}$ W. J. Johnson and A. Barone, J. Appl. Phys. 41, 2958 (1970).

${ }^{18}$ A. C. Scott, in Stochastic Behavior in Classical and Quantum Hamiltonian Systems, edited by G. Casati and J. Ford, Lecture notes in Physics (Springer, New York, 1979). 